\title{
Does Case Management Provide Support for Staff Facing Frequent Users of Emergency Departments? A Comparative Mixed-Method Evaluation of ED Staff Perception
}

\author{
Michael von Allmen ${ }^{1 *}$, Véronique S. Grazioli', Miriam Kasztura', Oriane Chastonay ${ }^{1}$, Joanna C. Moullin²,
} Olivier Hugli ${ }^{3}$, Jean-Bernard Daeppen ${ }^{4}$ and Patrick Bodenmann ${ }^{1}$

\begin{abstract}
Objective: Frequent users of emergency departments (FUED) account for a disproportionate number of emergency department (ED) visits and contribute to a wide range of challenges for ED staff. While several research has documented that case management (CM) tailored to FUED leads to a reduction in ED visits and a better quality of life (QoL) among FUED, whether there is added value for ED staff remains to be explored. This study aimed to compare, among staff in two academic EDs in Switzerland (one with and one without CM), the FUED-related knowledge, perceptions of the extent of the FUED issue, FUED-related work challenges and FUEDs' legitimacy to use ED.

Method: Mixed methods were employed. First, ED physicians and nurses $(N=253)$ of the two EDs completed an online survey assessing their knowledge and perceptions of FUEDs. Results between healthcare providers working in an ED with CM to those working in an ED without CM were compared using independent two-sided T-tests. Next, a sample of participants $(n=16)$ took part in a qualitative assessment via one-to-one interviews $(n=6)$ or focus groups $(n=10)$.

Results: Both quantitative and qualitative results documented that the FUED-related knowledge, the extent FUED were perceived as an issue and perceived FUEDs' legitimacy to use ED were not different between groups. The level of perceived FUED-related challenges was also similar between groups. Quantitative results showed that nurses with CM experienced more challenges related to FUED. Qualitative exploration revealed that lack of psychiatric staff within the emergency team and lack of communication between ED staff and CM team were some of the explanations behind these counterintuitive findings.
\end{abstract}

\footnotetext{
* Correspondence: michael.von.allmen@hotmail.ch

'Department of Vulnerabilities and Social Medicine, University Center for General Medicine and Public Health, Lausanne, Switzerland

Full list of author information is available at the end of the article
}

(c) The Author(s). 2021 Open Access This article is licensed under a Creative Commons Attribution 4.0 International License, which permits use, sharing, adaptation, distribution and reproduction in any medium or format, as long as you give appropriate credit to the original author(s) and the source, provide a link to the Creative Commons licence, and indicate if changes were made. The images or other third party material in this article are included in the article's Creative Commons licence, unless indicated otherwise in a credit line to the material. If material is not included in the article's Creative Commons licence and your intended use is not permitted by statutory regulation or exceeds the permitted use, you will need to obtain permission directly from the copyright holder. To view a copy of this licence, visit http://creativecommons.org/licenses/by/4.0/. The Creative Commons Public Domain Dedication waiver (http://creativecommons.org/publicdomain/zero/1.0/) applies to the data made available in this article, unless otherwise stated in a credit line to the data. 
Conclusion: Despite promising results on FUEDs' QoL and frequency of ED visits, these preliminary findings suggest that CM may provide limited support to ED staff in its current form. Given the high burden of FUED-related challenges encountered by ED staff, improved communication and FUED-related knowledge transfer between ED staff and the CM team should be prioritized to increase the value of a FUED CM intervention for ED staff.

Keywords: Frequent users of emergency departments, Case management, Emergency service, Staff

\section{Background}

Frequent users of emergency departments (FUED) have been the focus of increasing attention over the past decade. The term FUED refers to people who visit the emergency department (ED) 5 or more times in a 12month-period. They account for 4 to $16 \%$ of total ED users and 12 to $47 \%$ of ED visits, contributing to ED overcrowding and increasing health care costs [1-3].

FUED are a heterogenous group of patients sharing common characteristics [4]. Compared to ED patients who do not fulfil the FUED criteria, FUED have a higher prevalence of somatic and psychiatric comorbidities, psychological conditions, addiction $[1,5]$ and social issues $[1,6]$. They often cumulate vulnerabilities [7] leading to a higher mortality rate [8], and a poorer quality of life (QoL) [9]. Furthermore, FUED are likely to report feelings of discrimination, increasing their risk of being in situation of vulnerability [10].

In response, significant research efforts have been dedicated to develop interventions tailored to FUED, such as case management $(\mathrm{CM})$ [11]. CM oriented to FUED is a process conducted by health professionals (i.e., nurse, physician, social workers) inside and outside the $E D$, once any urgent issues have been solved. It aims to empower patients and increase their ability to interact with the healthcare system [12]. Published literature indicates that $\mathrm{CM}$ generally leads to a reduction in ED visits and healthcare costs [2,11-13]. Besides, it also improves FUEDs' QoL [9].

Surprisingly, there is very limited exploration regarding ED staff experiences caring for FUED. We are aware of only two qualitative studies involving ED staff on this topic, conducted in the USA and Singapore [14, 15]. Both studies report that staff faced challenges in addressing FUEDs' needs and experienced feelings of fatigue, failure and reduced mood. ED staff have a high prevalence of burnout [16], and any potential cause needs to be investigated. CM may alleviate these challenges. To our knowledge however, no study has explored whether there is added value for ED staff caring for FUED.

Therefore, this study was designed to address this gap in the literature. It aims at comparing FUED-related knowledge, the perception of the extent of FUED issue, perceived work challenges related to FUED and the perceived legitimacy of FUED ED visits between ED staff working in two academic ED only 45 miles apart, one with a nine-years' experience of $\mathrm{CM}$ implemented and one without it.

This study was nested in a larger ongoing research project that aimed to develop and implement a $\mathrm{CM}$ intervention tailored to FUEDs in the public hospitals with ED in the French-speaking region of Switzerland (project number 2018-00442) [17].

\section{Method \\ ED hospitals}

Research was conducted in two Swiss university hospitals $(45,000$ [with CM] and 75,000 [without CM] annual consultations). $\mathrm{CM}$ is an on-demand intervention provided by an external consultation team once a FUED is identified by the ED team. Also of note, the ED without $\mathrm{CM}$ has an integrated psychiatric emergency unit, whilst this is an external consultation service in the ED with CM.

\section{Quantitative methods \\ Sample}

Participants $(N=253)$ were ED staff working in these two Swiss university hospitals, divided by staff with $\mathrm{CM}$ $(n=100)$ and staff without CM $(n=153)$.

\section{Measures}

A 12-item online survey was developed to measure variables related to the FUED issue, summarized below and presented in Appendix 1. The survey was based on a version developed by a panel of experts involved with FUED and used in ongoing research [18, 19]. As described in Chastonay et al. [19], the panel conducted a series of sessions to develop a set of items exploring ED staff's perceptions regarding FUED and associated issues. The survey was tested by ED staff (Lausanne university hospital, CHUV; $N=14$ ). The version used in this study was composed by a selection of original items matching with its variables $[18,19]$.

Demographic variables The online survey included demographic variables (i.e., age, sex, years of practical experience and profession).

Dependent variables Participants were asked to indicate the extent to which they agreed with statements 
related to FUED. First, a statement explored the participants' own perception of their FUED-related knowledge. Next, their actual FUED- related knowledge was explored by assessment of their knowledge of FUEDs' attributes as reported in literature $[1,5,6]$. Then, statements explored the extent FUED are perceived as an issue, perceived level of FUED ED visit, perceived legitimacy of FUED ED visits and perception of FUED-related challenges (i.e., feeling of burnout, feeling of helplessness, organizational issues and FUED characteristics). (See Table 1).

Independent variables Type of emergency care (with/ without $\mathrm{CM}$ ) served as the independent variable (hereafter referred as groups), whereas sex (male/female), profession (nurse/ physician) and years of practice (0-6 years $1>6$ years, 6 years being the median) were used to stratify the analysis (hereafter referred as subgroups).

\section{Procedures}

From July 2018 to September 2018, all ED nurses and physicians of both hospitals were invited to complete the online survey. Email reminders were sent until at least a $60 \%$ [20] participation rate was achieved in both groups. All procedures were approved by the Swiss Ethics Committee (project number 2018-00442) [17].

\section{Analyses}

First, two-sided independent samples t-test were conducted with SPSS 25 to compare perceptions of FUED (i.e., dependent variables) between the groups with or without CM. Then, stratification was conducted by further $\mathrm{t}$-tests in subgroups (i.e., independent variables). The significance level was set at $p=.05$.

\section{FUED-related challenges}

The 16 variables regarding FUED-related challenges were subject to a principal component analysis (PCA). Suitability of data for factorial analysis was supported by correlation matrix inspection revealing coefficients of 0.3 and above, value of Kasier-Meyer-Olkin (0.87) and statistical significance of Bartlett's Test of Sphericity. PCA revealed the presence of four components with eigenvalues exceeding one, explaining 37.1, 10.2, 9.2, 6.8\% of the variance. Accordingly, a four-component solution consistent to literature $[1,5,6]$ was selected (hereafter referred as feeling of helplessness, organizational issues, FUED characteristics and feeling of burnout).

\section{Qualitative methods \\ Sample}

ED Nurses and physicians of both hospitals have received an email invitation to participate. Among them, 16 professionals were showed interest in participate and were included (with $(n=6)$ and without $(n=10) \mathrm{CM})$.

\section{Measures}

A grid of open-ended questions (see Appendix 2) was developed and employed in semi-structured interviews and focus groups to explore the perceived level of knowledge regarding FUED, extent FUED are perceived as an issue, perceived level FUED visit the ED, perceived legitimacy of FUED ED visits and perception of FUEDrelated challenges.

\section{Procedures}

Qualitative exploration regarding nurses was done through two focus groups (60 min each) by two study authors (MvA, VG), one with nurses working in the ED with $\mathrm{CM}(n=3)$ and the other with those in the ED

Table 1 Dependent variables

\begin{tabular}{|c|c|}
\hline Dependent variable & Measurement $^{c}$ \\
\hline \multicolumn{2}{|l|}{ FUED-related knowledge } \\
\hline level of perceived knowledge & 4-point Likert-type scale statement \\
\hline actual knowledge score & $\begin{array}{l}\text { Agreement mean score of fifteen 10-point Likert-type scale statements describing FUED characteristics supported } \\
\text { by existing evidence [17] (see appendix question 11) }\end{array}$ \\
\hline $\begin{array}{l}\text { Extent FUED are perceived as an } \\
\text { issue }\end{array}$ & 4-point Likert-type scale statement \\
\hline Perceived level of FUED ED visit & 4-point Likert-type scale statement \\
\hline $\begin{array}{l}\text { Perceived legitimacy of FUED ED } \\
\text { visits FUED }\end{array}$ & 10-pointl Likert-type scale statement \\
\hline $\begin{array}{l}\text { FUED-related work challenges: } \\
\text {-Feeling of burnout }\end{array}$ & $\begin{array}{l}\text { Sixteen 10-point Likert-type scale statements based on known FUED-related work challenges [18] (see appendix } \\
\text { question 8) summarize in } 4 \text { dependent variables after a principal component analysis (see data management) }\end{array}$ \\
\hline \multicolumn{2}{|l|}{-Feeling of helplessness } \\
\hline \multicolumn{2}{|l|}{-Organizational issues } \\
\hline -FUED characteristics & \\
\hline
\end{tabular}


without CM $(n=7)$. Qualitative assessment for physicians from EDs with CM $(n=3)$ and without CM $(n=3)$ was done through semi-structured interviews (20-45 min, conducted by MvA), since focus groups were not possible due to physicians's agenda contraints. Conversations were recorded after receiving participants' informed consent.

\section{Data management and analysis plan}

Interviews records were transcribed verbatim. Conventional content analysis was conducted on Atlas. Ti version 7 [21]. Initial coding was conducted by study authors (MvA, VG) using a line-by-line technique, whereby coders narrated the actions occurring in the interviews $[22,23]$. Following independent initial coding, a codebook was created in consensus meetings, pooling codes and eliminating idiosyncratic or redundant ones. Next, we used the codebook to independently doublecode $10 \%$ of the interviews until adequate intercoder consistency $(80 \%)$ was attained $[22,23]$. Once adequate intercoder consistency was established, the remaining interviews were coded independently by MvA.

\section{Results}

\section{Quantitative results}

In total, 296 participants completed the survey $(60 \%$ in the total staff of both hospitals). Of those, $85.5 \%$ completed more than demographic questions in the survey (i.e., information regarding age, sex, years of practical experience and profession), resulting in a final sample of 253 participants. Table 2 presents demographics by groups (CM, no-CM). Table 3 (c.f additional materials) presents descriptive statistics and t-tests results by groups (CM, no-CM) and within subgroups (physicians, nurses, males, females, 1-6 years of experience, $>6$ years of experience).

\section{Demographic results}

Participants were predominately female (67.6\%), reflecting the current proportion among health professionals

Table 2 Demographics results

\begin{tabular}{|c|c|c|c|c|c|}
\hline & \multicolumn{2}{|l|}{ CM } & \multicolumn{2}{|c|}{ No-CM } & \multirow[t]{2}{*}{$x^{2}$} \\
\hline & $S$ & $\%$ & $S$ & $\%$ & \\
\hline Gender & & & & & .070 \\
\hline Female & 61 & 61 & 110 & 71.9 & \\
\hline Male & 39 & 39 & 43 & 28.1 & \\
\hline Professions & & & & & .779 \\
\hline Physician & 31 & 31 & 50 & 32.7 & \\
\hline \multirow[t]{2}{*}{ Nurses } & 69 & 69 & 103 & 67.3 & \\
\hline & CM & No-CM & \multicolumn{2}{|c|}{ Statistics } & $P$-value \\
\hline Years of practical experience & 7.14 & 9.83 & \multicolumn{2}{|c|}{$t(250)=-2.741$} & .007 \\
\hline
\end{tabular}

in Switzerland [24]. Of the overall sample, 32\% were physicians and 68\% were either nurses or nurse assistants. Years of practical experience median was 6 years $(I Q R=10)$ and $67.2 \%$ were between 30 to 49 years old.

\section{Perceived level of knowledge and knowledge score}

Overall, the group with CM perceived their knowledge of FUED as significantly better than those working in a ED without CM. In subgroup analyses of ED staff with less work experience, their perception of their FUED knowledge was better in those with $\mathrm{CM}$ compare to those without. However, the actual knowledge score regarding FUED characteristics was not significantly different between groups and subgroups.

\section{Extent of the FUED issue}

Although it was not significantly different between groups, the physician subgroup with CM saw FUED as less of an issue than physicians without CM.

\section{Perceived level FUED visit the ED}

There was no significant difference in the perceived level of FUEDs' ED use between groups and subgroups.

\section{Perceived legitimacy of FUEDs' ED visits}

Legitimacy was not rated differently between groups. However, the more experienced healthcare provider subgroup in the ED with CM were more prone to consider FUED less legitimate to consult ED compared to those without it.

\section{Perception of FUED-related challenges}

Whereas perception of most challenges (i.e., feeling of burnout, organizational issues, FUED characteristics and feeling of helplessness) was not significantly different between groups, helplessness scores were significantly higher in nurses with than in those without CM.

\section{Qualitative results}

Participants $(N=16)$ were predominately females (87.5\%). Of the overall sample, $37.5 \%$ were physicians and $62,5 \%$ were nurses. Of physicians, $83 \%$ were chief residents and $17 \%$ senior physician certified in emergency medicine. Nurses' years of practical experience median was 9 years $(I Q R=9)$. Content analysis identified five main themes. Original quotes in French and translated in English are presented in Appendix 3.

\section{General knowledge of the FUED population and their characteristics}

General knowledge of FUED was considered insufficient among participants with and without CM (e.g., physician 3, no-CM: "It is not a population we are informed about. I have heard very little of recurrent ED patients as a 
Table 3 Results of t-tests and descriptive statistics by hospital (CM/ no CM) and within subgroups

\begin{tabular}{|c|c|c|c|c|c|c|c|c|c|c|c|c|c|c|}
\hline \multirow[t]{2}{*}{ Variable } & \multirow{2}{*}{\multicolumn{2}{|c|}{$\frac{\text { All }}{\text { CM }}$}} & \multirow{2}{*}{$\begin{array}{l}\text { Physicians } \\
\text { No CM }\end{array}$} & \multicolumn{3}{|c|}{ Nurses } & \multicolumn{2}{|l|}{ Male } & \multicolumn{2}{|c|}{ Female } & \multicolumn{2}{|c|}{$1-6$ y. of exp. } & \multicolumn{2}{|c|}{$>6$ y. of exp. } \\
\hline & & & & \multicolumn{3}{|l|}{ CM } & \multicolumn{2}{|c|}{ No CM } & \multicolumn{2}{|l|}{ CM } & \multicolumn{2}{|c|}{ No CM } & \multicolumn{2}{|l|}{ CM } \\
\hline \multicolumn{15}{|c|}{ 1. Level of perceived knowledge } \\
\hline$M(S D)$ & $\begin{array}{l}2.63 \\
(.726)\end{array}$ & $\begin{array}{l}2.83 \\
(.65)\end{array}$ & $2.62(.82)$ & $\begin{array}{l}2.81 \\
(.68)\end{array}$ & $\begin{array}{l}2.63 \\
(.689)\end{array}$ & $\begin{array}{l}2.84 \\
(.641)\end{array}$ & $\begin{array}{l}2.43 \\
(.765)\end{array}$ & $\begin{array}{l}2.73 \\
(.679)\end{array}$ & $\begin{array}{l}2.75 \\
(.68)\end{array}$ & $\begin{array}{l}2.87 \\
(.64)\end{array}$ & $\begin{array}{l}2.72 \\
(.64)\end{array}$ & $\begin{array}{l}2.97 \\
(.605)\end{array}$ & $\begin{array}{l}2.49 \\
(.82)\end{array}$ & $\begin{array}{l}2.74 \\
(.67)\end{array}$ \\
\hline $\mathrm{n}$ & 142 & 97 & 47 & 29 & 68 & 95 & 40 & 37 & 60 & 102 & 58 & 61 & 39 & 80 \\
\hline $95 \% \mathrm{Cl}$ & \multicolumn{2}{|c|}{$.021-.383$} & \multicolumn{2}{|l|}{$-.154-.534$} & \multicolumn{2}{|c|}{$-.001-.420$} & \multicolumn{2}{|c|}{$-.035-.620$} & \multicolumn{2}{|c|}{$-.088-.333$} & \multicolumn{2}{|c|}{$.016-.470$} & \multicolumn{2}{|c|}{$-.053-.553$} \\
\hline $\mathrm{t}$ & \multicolumn{2}{|l|}{$2.2^{*}$} & 1.08 & & 1.97 & & 1.77 & & 1.15 & & $2.12^{*}$ & & 1.65 & \\
\hline 2. Actual & knowlec & e score & & & & & & & & & & & & \\
\hline M (SD) & $\begin{array}{l}6.50 \\
(1.39)\end{array}$ & $\begin{array}{l}6.61 \\
(1.51)\end{array}$ & $6.67(1.18)$ & $\begin{array}{l}6.87 \\
(1.29)\end{array}$ & $\begin{array}{l}6.58 \\
(1.48)\end{array}$ & $\begin{array}{l}6.47 \\
(1.6)\end{array}$ & $\begin{array}{l}6.67 \\
(1.37)\end{array}$ & $\begin{array}{l}6.38 \\
(1.23)\end{array}$ & $\begin{array}{l}6.56 \\
(1.4)\end{array}$ & $\begin{array}{l}6.69 \\
(1.61)\end{array}$ & $\begin{array}{l}6.47 \\
(1.41)\end{array}$ & $\begin{array}{l}6.47 \\
(1.54)\end{array}$ & $\begin{array}{l}6.8 \\
(1.35)\end{array}$ & $\begin{array}{l}6.7 \\
(1.46)\end{array}$ \\
\hline$n$ & 96 & 142 & 29 & 47 & 67 & 95 & 37 & 40 & 60 & 102 & 58 & 61 & 39 & 80 \\
\hline $95 \% \mathrm{Cl}$ & $-.378-$ & & $-.377-.799$ & & $-.592-$ & & -.877 & & -.365 & & $-.530-$ & & -.700 & \\
\hline $\mathrm{t}$ & .018 & & .714 & & -.415 & & .952 & & $(159)=$ & & .026 & & -.513 & \\
\hline 3. Extent & FUED ar & perceive & as an issue & & & & & & & & & & & \\
\hline M (SD) & $\begin{array}{l}2.49 \\
(.703)\end{array}$ & $\begin{array}{l}2.57 \\
(.572)\end{array}$ & $2.19(.703)$ & $\begin{array}{l}2.54 \\
(.544)\end{array}$ & $\begin{array}{l}2.62 \\
(.66)\end{array}$ & $\begin{array}{l}2.58 \\
(.586\end{array}$ & $\begin{array}{l}2.46 \\
(.79)\end{array}$ & $\begin{array}{l}2.53 \\
(.550)\end{array}$ & $\begin{array}{l}2.51 \\
(.649)\end{array}$ & $\begin{array}{l}2.58 \\
(.582)\end{array}$ & $\begin{array}{l}2.46 \\
(.721)\end{array}$ & $\begin{array}{l}2.57 \\
(.558)\end{array}$ & $\begin{array}{l}2.54 \\
(.682)\end{array}$ & $\begin{array}{l}2.58 \\
(.585)\end{array}$ \\
\hline$n$ & 100 & 151 & 31 & 48 & 69 & 103 & 39 & 43 & 61 & 108 & 61 & 65 & 39 & 85 \\
\hline $95 \% \mathrm{Cl}$ & $-.087-$ & & $.068-.629$ & & $-.231-$ & & -.229 & & -.117 & & $-.118-$ & & -.198 & \\
\hline t & .943 & & $2.47^{*}$ & & -.422 & & .483 & & .773 & & .956 & & .219 & \\
\hline 4. Perceiv & red level & $f$ FUED & visit & & & & & & & & & & & \\
\hline M (SD) & $\begin{array}{l}2.18 \\
(.757)\end{array}$ & $\begin{array}{l}2.17 \\
(.725)\end{array}$ & $2.16(.638)$ & $\begin{array}{l}2.29 \\
(.771)\end{array}$ & $\begin{array}{l}2.19 \\
(.809)\end{array}$ & $\begin{array}{l}2.11 \\
(.699)\end{array}$ & $\begin{array}{l}2.08 \\
(664)\end{array}$ & $\begin{array}{l}2.40 \\
(.849)\end{array}$ & $\begin{array}{l}2.25 \\
(.809)\end{array}$ & $\begin{array}{l}2.07 \\
(.651)\end{array}$ & $\begin{array}{l}2.25 \\
(.789)\end{array}$ & $\begin{array}{l}2.32 \\
(.773)\end{array}$ & $\begin{array}{l}2.08 \\
(.703)\end{array}$ & $\begin{array}{l}2.05 \\
(.671)\end{array}$ \\
\hline$n$ & 100 & 151 & 31 & 48 & 69 & 103 & 39 & 43 & 61 & 108 & 61 & 65 & 39 & 85 \\
\hline $95 \% \mathrm{Cl}$ & $-.202-$ & & $-.201-.462$ & & $-.310-$ & & -.019 & & -.412 & & $-.198-$ & & -.291 & \\
\hline t & -.152 & & .435 & & -.704 & & 1.88 & & -1.42 & & .555 & & .821 & \\
\hline 5. Perceiv & ed legiti & lacy of & ED ED visits & & & & & & & & & & & \\
\hline M (SD) & $\begin{array}{l}4.22 \\
(2.34)\end{array}$ & $\begin{array}{l}3.89 \\
(2.23)\end{array}$ & $4.07(1.86)$ & $\begin{array}{l}3.70 \\
(2.28)\end{array}$ & $\begin{array}{l}4.29 \\
(2.53)\end{array}$ & $\begin{array}{l}3.98 \\
(2.20)\end{array}$ & $\begin{array}{l}4.16 \\
(2.48)\end{array}$ & $\begin{array}{l}3.81 \\
(2.45)\end{array}$ & $\begin{array}{l}4.26 \\
(2.26)\end{array}$ & $\begin{array}{l}3.92 \\
(2.14)\end{array}$ & $\begin{array}{l}3.7 \\
(2.03)\end{array}$ & $\begin{array}{l}3.94 \\
(2.3)\end{array}$ & $\begin{array}{l}5.05 \\
(2.58)\end{array}$ & $\begin{array}{l}3.86 \\
(2.18)\end{array}$ \\
\hline$n$ & 99 & 146 & 30 & 47 & 69 & 99 & 38 & 42 & 61 & 104 & 61 & 63 & 38 & 83 \\
\hline $95 \% \mathrm{Cl}$ & $-.912-$ & & $-1.335-.626$ & & $-1.03-$ & & $-1.45-$ & & -1.03 & & $-.540-$ & 003 & -2.09 & -300 \\
\hline $\mathrm{t}$ & -1.12 & & -.733 & & -.844 & & -.632 & & -.962 & & .594 & & -2.64 & \\
\hline 6. Percep & tion of $\mathrm{F}$ & ED-relat & challenges: & Feeling & burnout & & & & & & & & & \\
\hline M (SD) & $\begin{array}{l}6.63 \\
(1.83)\end{array}$ & $\begin{array}{l}6.70 \\
(2.13)\end{array}$ & $6.32(1.87)$ & $\begin{array}{l}6.48 \\
(1.74)\end{array}$ & $\begin{array}{l}2.76 \\
(1.80)\end{array}$ & $\begin{array}{l}6.80 \\
(2.29)\end{array}$ & $\begin{array}{l}6.61 \\
(1.76)\end{array}$ & $\begin{array}{l}6.14 \\
(1.97)\end{array}$ & $\begin{array}{l}6.63 \\
(1.89)\end{array}$ & $\begin{array}{l}6.92 \\
(2.16)\end{array}$ & $\begin{array}{l}6.82 \\
(1.86)\end{array}$ & $\begin{array}{l}6.63 \\
(1.95)\end{array}$ & $\begin{array}{l}6.33 \\
(1.78)\end{array}$ & $\begin{array}{l}6.74 \\
(2.28)\end{array}$ \\
\hline$n$ & 100 & 151 & 31 & 48 & 69 & 103 & 39 & 43 & 61 & 108 & 61 & 65 & 39 & 85 \\
\hline $95 \% \mathrm{Cl}$ & $-.44-.5$ & & $-.67-.98$ & & $-.58-.6$ & & -1.29 & & $-.37-$ & & $-.86-.4$ & & $-.41-$ & \\
\hline $\mathrm{t}$ & .276 & & .378 & & .120 & & -1.15 & & .863 & & -.556 & & 1.079 & \\
\hline 7. Percep & tion of $\mathrm{F}$ & ED-relat & challenges: & Feeling & helpless & ness ${ }^{b}$ & & & & & & & & \\
\hline$M(S D)$ & $\begin{array}{l}6.63 \\
(2.15)\end{array}$ & $\begin{array}{l}5.95 \\
(2.35)\end{array}$ & $5.98(2.36)$ & $\begin{array}{l}6.37 \\
(2.15)\end{array}$ & $\begin{array}{l}6.93 \\
(1.99)\end{array}$ & $\begin{array}{l}5.76 \\
(2.42)\end{array}$ & $\begin{array}{l}6.34 \\
(2.02)\end{array}$ & $\begin{array}{l}5.83 \\
(2.50)\end{array}$ & $\begin{array}{l}6.82 \\
(2.22)\end{array}$ & $\begin{array}{l}6.01 \\
(2.30)\end{array}$ & $\begin{array}{l}6.51 \\
(2.23)\end{array}$ & $\begin{array}{l}6.00 \\
(2.14)\end{array}$ & $\begin{array}{l}6.82 \\
(2.02)\end{array}$ & $\begin{array}{l}5.99 \\
(2.44)\end{array}$ \\
\hline$n$ & 100 & 151 & 31 & 48 & 69 & 103 & 39 & 43 & 61 & 108 & 61 & 65 & 39 & 85 \\
\hline $95 \% \mathrm{Cl}$ & $-1.25-$ & & $-.63-1.42$ & & $-1.86-$ & 4.72 & -1.52 & & -1.53 & 0.095 & -1.28 & & -1.71 & \\
\hline $\mathrm{t}$ & -2.3 & & .768 & & $-3.3^{* *}$ & & -1.01 & & -2.23 & & -1.32 & & -1.85 & \\
\hline 8. Percep & tion of $\mathrm{F}$ & ED-relat & challenges: & Organiz & onal issu & & & & & & & & & \\
\hline M (SD) & $\begin{array}{l}6.6 \\
(2.11)\end{array}$ & $\begin{array}{l}6.75 \\
(2.14)\end{array}$ & $6.46(2.29)$ & $\begin{array}{l}7.37 \\
(2.08)\end{array}$ & $\begin{array}{l}6.67 \\
(2.04)\end{array}$ & $\begin{array}{l}6.46 \\
(2.12)\end{array}$ & $\begin{array}{l}6.81 \\
(1.86)\end{array}$ & $\begin{array}{l}6.87 \\
(2.32)\end{array}$ & $\begin{array}{l}6.47 \\
(2.26)\end{array}$ & $\begin{array}{l}6.70 \\
(2.07)\end{array}$ & $\begin{array}{l}6.69 \\
(2.01)\end{array}$ & $\begin{array}{l}6.80 \\
(1.84)\end{array}$ & $\begin{array}{l}6.47 \\
(2.28)\end{array}$ & $\begin{array}{l}6.71 \\
(2.37)\end{array}$ \\
\hline$n$ & 100 & 148 & 31 & 48 & 69 & 100 & 39 & 43 & 61 & 105 & 61 & 65 & 39 & 89 \\
\hline
\end{tabular}


Table 3 Results of t-tests and descriptive statistics by hospital (CM/ no CM) and within subgroups (Continued)

\begin{tabular}{|c|c|c|c|c|c|c|c|c|c|c|c|c|c|c|}
\hline \multirow[t]{2}{*}{ Variable } & \multicolumn{2}{|l|}{ All } & \multirow{2}{*}{$\begin{array}{l}\text { Physicians } \\
\text { No CM }\end{array}$} & \multicolumn{3}{|c|}{ Nurses } & \multicolumn{2}{|l|}{ Male } & \multicolumn{2}{|c|}{ Female } & \multicolumn{2}{|c|}{ 1-6 y. of exp. } & \multicolumn{2}{|c|}{$>6 \mathrm{y}$. of exp. } \\
\hline & \multicolumn{2}{|l|}{$\mathrm{CM}$} & & \multicolumn{3}{|l|}{$\mathrm{CM}$} & \multicolumn{2}{|c|}{ No CM } & \multicolumn{2}{|l|}{$\mathrm{CM}$} & \multicolumn{2}{|c|}{ No CM } & \multicolumn{2}{|c|}{$\mathrm{CM}$} \\
\hline $95 \% \mathrm{Cl}$ & \multicolumn{2}{|c|}{$-.39-.69$} & \multicolumn{2}{|l|}{$-.096-1.89$} & \multicolumn{2}{|c|}{$-.85-.44$} & \multicolumn{2}{|c|}{$-.87-.99$} & \multicolumn{2}{|c|}{$-.45-.91$} & \multicolumn{2}{|c|}{$.12-.344$} & \multicolumn{2}{|c|}{$.232-.456$} \\
\hline t & \multicolumn{2}{|l|}{.538} & \multicolumn{2}{|l|}{1.79} & \multicolumn{2}{|c|}{-.632} & \multicolumn{2}{|c|}{.138} & \multicolumn{2}{|l|}{.664} & \multicolumn{2}{|l|}{.346} & \multicolumn{2}{|l|}{.511} \\
\hline \multicolumn{15}{|c|}{ 9. Perception of FUED-related challenges: FUED characteristics ${ }^{\mathrm{a}}$} \\
\hline M (SD) & $\begin{array}{l}6.88 \\
(1.45)\end{array}$ & $\begin{array}{l}6.73 \\
(1.76)\end{array}$ & $7.06(1.39)$ & $\begin{array}{l}6.96 \\
(1.54)\end{array}$ & $\begin{array}{l}6.80 \\
(1.48)\end{array}$ & $\begin{array}{l}6.61 \\
(1.85)\end{array}$ & $\begin{array}{l}7.01 \\
(1.30)\end{array}$ & $\begin{array}{l}6.36 \\
(1.93)\end{array}$ & $\begin{array}{l}6.80 \\
(1.54)\end{array}$ & $\begin{array}{l}6.87 \\
(1.67)\end{array}$ & $\begin{array}{l}6.88 \\
(1.45)\end{array}$ & $\begin{array}{l}6.84 \\
(1.61)\end{array}$ & $\begin{array}{l}6.89 \\
(1.46)\end{array}$ & $\begin{array}{l}6.67 \\
(1.86)\end{array}$ \\
\hline$n$ & 100 & 151 & 31 & 48 & 69 & 103 & 39 & 43 & 61 & 108 & 61 & 65 & 39 & 85 \\
\hline $95 \% \mathrm{Cl}$ & \multicolumn{2}{|c|}{$-.57-.26$} & \multicolumn{2}{|l|}{$-.78-.58$} & \multicolumn{2}{|c|}{$-.71-.34$} & \multicolumn{2}{|c|}{$-1.38-.08$} & \multicolumn{2}{|c|}{$-.44-.59$} & \multicolumn{2}{|c|}{$-.58-.50$} & \multicolumn{2}{|c|}{$-.88-.45$} \\
\hline t & \multicolumn{2}{|l|}{-.732} & -.290 & & -.692 & & -1.77 & & .281 & & -.141 & & -.648 & \\
\hline
\end{tabular}

${ }^{*} p<0.05,{ }^{* *} p<0.01,{ }^{* * *} p<0.001$

specific entity or type of patient that would require specific management").

\section{Extent of FUED issue}

Both groups reported they frequently encountered FUED (e.g., physician 3, no-CM: "It is still important in terms of the number of patients and frequency of emergency room visits").

\section{FUED legitimacy to consult ED}

FUEDs' legitimacy to consult ED was considered equally low between participants with and without CM, due to absence of medical conditions justifying ED consultations (e.g, physician 1, CM: "the place for these people is not emergency rooms") (e.g., physician 2, no-CM: "It's people who are in good health (...) don't have many comorbidities").

\section{Challenges encountered in the management of FUED}

Participants with and without CM experienced the same range of challenges when providing healthcare to FUED (e.g., physician 3, CM:" The first thing in these patients is: time consuming, annoying and generates negative counter-transfers"; physician 2, CM: "We just can't heal them. So yes, it awakens a feeling of helplessness in the team and fatigue").

\section{Perceptions of FUEDs management, its strengths and weaknesses}

Participants in both groups saw numerous benefits of CM tailored to FUED, such as "adapting patient care to their needs and demands" or "coordinating FUEDs' healthcare network". Negative aspects of CM were predominantly raised in the nurse subgroup with CM. Reported issues were a lack of information and feedback regarding CM activities (Nurses' focus group, CM: "I wasn't aware that they were actually doing all this (...) we have less information on what the "vulnerable populations" team (i.e., CM team) do (...) We're potentially biased because it's suddenly patients we don't see anymore and we don't necessarily realize"). Furthermore, negative evaluation of psychiatric management for FUED was also pointed out (Nurses' focus group, CM: "When you see someone who comes in a recurring way (...) and a quarter of an hour after coming down from a psychiatric consultation, you can't say it's efficient or welldone care").

\section{Discussion and conclusion}

This study is the first quantitative and qualitative exploration of the potential perceived added-value of CM for ED staff, by comparing the perceptions of FUED by ED staff with and without a CM service.

Unexpectedly, in both quantitative and qualitative results, FUED-related knowledge was no better in CM group despite a higher subjective appreciation of it from physicians with CM. These findings suggest that CM for FUED does not contribute to a knowledge transfer to ED staff. To enhance this transfer, active learning approaches conducted by the CM team may be used (e.g., workshops or feed-back sessions on specific patients)." [25].

Hudon et al. found in a primary care setting that CM, by reducing the FUEDs' psychological distress, made caregivers feel more confident in dealing with FUED challenges [26]. In the ED setting, our results did not come to a similar conclusion. Paradoxically, quantitative results revealed a higher level of helplessness in nurses with CM. The hypothesis is that these results may pertain to confounding factors. First, profession discrepancy might be explained by the confounding effect of professional status (e.g., level of self-awareness and expectations of oneself, difficulty to admit lack of competency) [27]. Furthermore, management of psychiatric emergencies were quite different between the two EDs and may have confounded CM perception. The external psychiatrist consultation service in the ED with $\mathrm{CM}$ was subject to negative evaluation from nurses in qualitative exploration. An integrated psychiatric unit may provide greater support to staff facing FUED psychiatric and 
behavioral issues, representing an important part of FUED care [17]. In addition, qualitative exploration revealed that $\mathrm{CM}$ activity was considered not visible enough. Specifically, participants highlighted a lack of feedback from the CM team concerning referred FUED. The insufficient communication between $\mathrm{CM}$ and $\mathrm{ED}$ teams prevented ED staff from being informed of the $\mathrm{CM}$ team's successes and failures. A better communication between ED and CM team may help address ED staff's feeling of helplessness.

$\mathrm{CM}$ has been proven to reduce FUED consultations in ED [17]. However, the perceived level FUED visit the ED was not quantitatively different between groups. That said, physicians with $\mathrm{CM}$ tended to perceive FUED as less of an issue compared to those without. This may also pertain to the fact that nurses and physician are not exposed to FUED-challenges in the same way.

Perception of FUEDs' legitimacy to use ED did not appear to be impacted by CM implementation. Surprisingly, the qualitative analysis revealed that participants in both groups considered FUEDs' ED visits as inappropriate. This does not match reality, as most FUEDs' visits are triggered by objective acute healthcare needs [1,28]. .Studies conducted in psychiatry demonstrate that staff knowledge is an important factor to foster empathy towards a stigmatized population [29]. The general lack of FUED-related knowledge may explain why both groups perceived FUED to lack legitimacy. Increasing the knowledge transfer through $\mathrm{CM}$ team might also address FUEDs' perception of discrimination.

This study has several limitations. First, the quantitative survey was not previously validated beyond face validity, although it was used in a previous studies $[18,19]$. That being said, the survey development went through an expert committee and iterative testing. Second, the study design did not allow for the control of confounding factors. However, triangulation of quantitative and qualitative data strengthened the validity of the analysis. Generalizability of data is also increased by the EDs studied (i.e., two out of five university EDs in Switzerland).

Although preliminary, our findings suggest two recommendations for allowing $\mathrm{CM}$ to address FUED challenges experienced by ED staff. First, good communication between ED staff and the CM team is important to support ED staff in their challenges to care for FUED; it contributes to knowledge transfer and eventually decrease perception of FUED illegitimacy to visit ED. Second, we recommend reinforcing collaboration between ED staff and psychiatrists to help address FUED care complexity, by adding a psychiatrist to the CM team if no psychiatry team is present in the ED.

To conclude, despite promising results on FUEDs' QoL and ED visits, CM may provide limited support to
ED staff in its current form. Given the high burden of FUED-related challenges encountered by ED staff, improved communication and FUED-related knowledge transfer between ED staff and the CM team should be prioritized to increase the CM added-value for ED staff.

\section{Abbreviations}

FUED: Frequent users of emergency department; ED: Emergency department; QoL: Quality of life; CM: Case management; PCA: principal component analyses

\section{Supplementary Information}

The online version contains supplementary material available at https://doi. org/10.1186/s12873-021-00481-9.

Additional file 1 .

Acknowledgements

N/A

Authors' contributions

MvA was responsible for data collection and analyses, supervised by VSG and PB. MvA was in charge of the manuscript writing, to which all authors (VSG, $\mathrm{PB}, \mathrm{MK}, \mathrm{JM}, \mathrm{OC}, \mathrm{OH}$ and JBD) have contributed. All authors read and approved the final manuscript.

\section{Funding}

This study had not beneficiated from financial support.

\section{Availability of data and materials}

The datasets used and/or analyzed during the current study are available from the corresponding author on reasonable request.

\section{Declarations}

Ethics approval and consent to participate

Ethical approval was obtained from ethics committee of CER-VD (the commission cantonale d'éthique de la recherche sur l'être humain) in relation to the research project number 2018-00442 which our study is nested in. The CERVD is the main IRB for the current project because it covers the Canton where the project is conducted (i.e., directed and coordinated). All procedures followed the Declaration of Helsinki. Participant's informed consent was obtained in each part (i.e., quantitative and qualitative) of the study.

Consent for publication

$\mathrm{N} / \mathrm{A}$

\section{Competing interests}

The authors declare that they have no competing interests.

\section{Author details}

${ }^{1}$ Department of Vulnerabilities and Social Medicine, University Center for General Medicine and Public Health, Lausanne, Switzerland. ${ }^{2}$ Faculty of Health Sciences, Curtin University, Perth, Australia. ${ }^{3}$ Emergency Department, University Hospital, Lausanne, Switzerland. ${ }^{4}$ Addiction Medicine, Department of Psychiatry, Lausanne University Hospital, University of Lausanne, Lausanne, Switzerland.

Received: 17 March 2021 Accepted: 5 July 2021

Published online: 04 August 2021

\section{References}

1. Giannouchos TV, Kum H-C, Foster MJ, Ohsfeldt RL. Characteristics and predictors of adult frequent emergency department users in the United States: a systematic literature review. J Eval Clin Pract. 2019;25(3):420-33. https://doi.org/10.1111/jep.13137. 
2. Hoot NR, Aronsky D. Systematic review of emergency department crowding: causes, effects, and solutions. Ann Emerg Med. 2008;52(2):126-36. https://doi.org/10.1016/j.annemergmed.2008.03.014.

3. Pines JM, Hilton JA, Weber EJ, Alkemade AJ, Al Shabanah H, Anderson PD, et al. International perspectives on emergency department crowding. Acad Emerg Med. 2011;18(12):1358-70. https://doi.org/10.1111/j.1553-2712.2011. 01235.x.

4. Slankamenac K, Zehnder M, Langner TO, Krähenmann K, Keller DI. Recurrent Emergency Department Users: Two Categories with Different Risk Profiles. J Clin Med. 2019:8(3):333. https://doi.org/10.3390/jcm8030333.

5. Vu F, Daeppen J-B, Hugli O, Iglesias K, Stucki S, Paroz S, et al. Screening of mental health and substance users in frequent users of a general Swiss emergency department. BMC Emergency Medicine. 2015;15(1):27. https:// doi.org/10.1186/s12873-015-0053-2.

6. Bieler G, Paroz S, Faouzi M, Trueb L, Vaucher P, Althaus F, et al. Social and medical vulnerability factors of emergency department frequent users in a universal health insurance system. Acad Emerg Med. 2012;19(1):63-8. https://doi.org/10.1111/j.1553-2712.2011.01246.x. Epub 2012 Jan 5.

7. Bodenmann P, Baggio S, Iglesias K, Althaus F, Velonaki V-S, Stucki S, et al. Characterizing the vulnerability of frequent emergency department users by applying a conceptual framework: a controlled, cross-sectional study. Int J Equity Health. 2015;14:146. https://doi.org/10.1186/s12939-015-0277-5.

8. Moe J, Kirkland S, Ospina MB, Campbell S, Long R, Davidson A, et al. Mortality, admission rates and outpatient use among frequent users of emergency departments: a systematic review. Emerg Med J. 2016;33(3):2306. https://doi.org/10.1136/emermed-2014-204496. Epub 2015 May 7.

9. Iglesias K, Baggio S, Moschetti K, Wasserfallen J-B, Hugli O, Daeppen J-B, et al. Using case management in a universal health coverage system to improve quality of life of frequent emergency department users: a randomized controlled trial. Qual Life Res. 2018;27(2):503-13. https://doi. org/10.1007/s11136-017-1739-6.

10. Baggio S, Iglesias K, Hugli O, Burnand B, Ruggeri O, Wasserfallen J-B, et al. Associations between perceived discrimination and health status among frequent emergency department users. Eur J Emerg Med. 2017;24(2):136-41. https://doi.org/10.1097/MEJ.0000000000000311.

11. Di Mauro R, Di Silvio V, Bosco P, Laquintana D, Galazzi A. Case management programs in emergency department to reduce frequent user visits: a systematic review. Acta Biomed. 2019;90(6-S):34-40.

12. Bodenmann P, Velonaki V-S, Griffin JL, Baggio S, Iglesias K, Moschetti K, et al. Case management may reduce emergency department frequent use in a universal health coverage system: a randomized controlled trial. J Gen Intern Med. 2017;32(5):508-15. https://doi.org/10.1007/s11606-016-3789-9.

13. Moe J, Kirkland SW, Rawe E, Ospina MB, Vandermeer B, Campbell S, et al. Effectiveness of interventions to decrease emergency department visits by adult frequent users: a systematic review. Acad Emerg Med. 2017 Jan 1; 24(1):40-52. https://doi.org/10.1111/acem.13060.

14. Malone RE. Almost 'like family': emergency nurses and 'frequent flyers'. J Emerg Nurs. 1996;22(3):176-83. https://doi.org/10.1016/S0099-1767(96)801 02-4.

15. Poremski D, Kunjithapatham G, Koh D, Lim XY, Alexander M, Lee C. Lost keys: understanding service Providers' impressions of frequent visitors to psychiatric emergency Services in Singapore. Psychiatr Serv. 2017;68(4):3905. https://doi.org/10.1176/appi.ps.201600165.

16. Moukarzel A, Michelet P, Durand A-C, Sebbane M, Bourgeois S, Markarian T, et al. Burnout syndrome among emergency department staff: prevalence and associated factors. Biomed Res Int. 2019;2019:6462472.

17. Grazioli VS, Moullin JC, Kasztura M, Canepa-Allen M, Hugli O, Griffin J, et al. Implementing a case management intervention for frequent users if the emergency department (I-CaM): an effectiveness-implementation hybrid trial study protocol. BMC Health Serv Res. 2019;19(1):28. https://doi.org/10.11 86/s12913-018-3852-9.

18. Grazioli VS, Kastura M, Chastonay O, Graells M, Schumtz E, von Allmen M, Lemoine M, Daeppen JB, Hugli O, Bodenmann P, Healthcare providers' perceptions of difficulties related to frequent users of emergency departments, INQUIRY: The Journal of Health Care Organization, Provision, and Financing (in process of publication)

19. Chastonay OJ, Lemoine M, Grazioli VS, Canepa Allen M, Kasztura M, Moullin $J C$, et al. Health care providers' perception of the frequent emergency department user issue and of targeted case management interventions: a cross-sectional national survey in Switzerland. BMC Emerg Med. 2021;21(1):4.
20. Livingston EH, Wislar JS. Minimum response rates for survey research. Arch Surg. 2012 Feb 20;147(2):110. https://doi.org/10.1001/archsurg.2011.2169.

21. Hsieh H-F, Shannon SE. Three approaches to qualitative content analysis. Qual Health Res. 2005;15(9):1277-88. https://doi.org/10.1177/10497323052 76687.

22. Miles MB, Huberman AM. Qualitative data analysis : An expanded sourcebook. Thousand Oaks: Sage publishing, Inc; 2005.

23. Shek DTL, Tang VMY, Han XY. Evaluation of evaluation studies using qualitative research methods in social literature (1990-2003): Evidence that constitutes a wake-up call. Res Soc Work Pract. 2005;15:180-94.

24. Statistiques médecins, Office fédérale de la santé publique OFSP. https:// www.bag.admin.ch/bag/fr/home/zahlen-und-statistiken/statistiken-berufeim-gesundheitswesen/statistiken-medizinalberufe1/statistiken-aerztinnen-a erzte.html

25. Pluta WJ, Richards BF, Mutnick A. PBL and Beyond: Trends in Collaborative Learning. Teach Learn Med. 2013;25(sup1):S9-16.

26. Hudon C, Chouinard M-C, Dubois M-F, Roberge P, Loignon C, Tchouaket É, et al. Case Management in Primary Care for frequent users of health care services: a mixed methods study. Ann Fam Med mai. 2018;16(3):232-9. https://doi.org/10.1370/afm.2233.

27. Casillas A, Paroz S, Green AR, Wolff H, Weber O, Faucherre F, et al. Cultural competency of health-care providers in a Swiss University Hospital: selfassessed cross-cultural skillfulness in a cross-sectional study. BMC Med Educ 2014;14(1):19.

28. Krieg C, Hudon C, Chouinard MC, Dufour I. Individual predictors of frequent emergency department use: a scoping review. BMC Health Serv Res. 2016; 16(1):594. https://doi.org/10.1186/s12913-016-1852-1.

29. Hinshaw SP, Stier A. Stigma as related to mental disorders. Annu Rev Clin Psychol. 2008;4(1):367-93. https://doi.org/10.1146/annurev.clinpsy.4.022 007.141245 .

\section{Publisher's Note}

Springer Nature remains neutral with regard to jurisdictional claims in published maps and institutional affiliations.

Ready to submit your research? Choose BMC and benefit from:

- fast, convenient online submission

- thorough peer review by experienced researchers in your field

- rapid publication on acceptance

- support for research data, including large and complex data types

- gold Open Access which fosters wider collaboration and increased citations

- maximum visibility for your research: over $100 \mathrm{M}$ website views per year

At $\mathrm{BMC}$, research is always in progress.

Learn more biomedcentral.com/submissions 